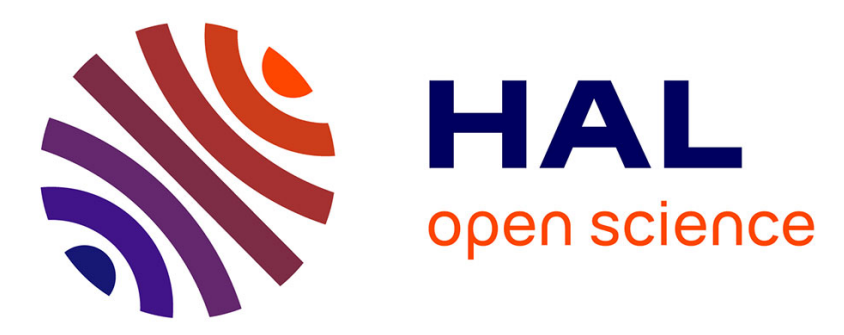

\title{
TELEPHONE MOBILE ET EXPRESSION IDENTITAIRE : REFLEXIONS SUR L'EXPOSITION TECHNOLOGIQUE DE SOI PARMI LES JEUNES
}

Nayra Vacaflor, Mahdi Amri

\section{- To cite this version:}

Nayra Vacaflor, Mahdi Amri. TELEPHONE MOBILE ET EXPRESSION IDENTITAIRE : REFLEXIONS SUR L'EXPOSITION TECHNOLOGIQUE DE SOI PARMI LES JEUNES. Les Enjeux de l'information et de la communication, 2010. hal-02480516

\author{
HAL Id: hal-02480516 \\ https://hal.science/hal-02480516
}

Submitted on 16 Feb 2020

HAL is a multi-disciplinary open access archive for the deposit and dissemination of scientific research documents, whether they are published or not. The documents may come from teaching and research institutions in France or abroad, or from public or private research centers.
L'archive ouverte pluridisciplinaire HAL, est destinée au dépôt et à la diffusion de documents scientifiques de niveau recherche, publiés ou non, émanant des établissements d'enseignement et de recherche français ou étrangers, des laboratoires publics ou privés. 


\title{
TÉLÉPHONE MOBILE ET EXPRESSION IDENTITAIRE : RÉFLEXIONS SUR L'EXPOSITION TECHNOLOGIQUE DE SOI PARMI LES JEUNES
}

\author{
Mahdi Amri et Nayra Vacaflor
}

GRESEC | « Les Enjeux de l'information et de la communication »

2010/1 Volume 2010 | pages 1 à 17

\section{Article disponible en ligne à l'adresse :}

https://www.cairn.info/revue-les-enjeux-de-l-information-et-de-lacommunication-2010-1-page-1.htm

Distribution électronique Cairn.info pour GRESEC.

(C) GRESEC. Tous droits réservés pour tous pays.

La reproduction ou représentation de cet article, notamment par photocopie, n'est autorisée que dans les limites des conditions générales d'utilisation du site ou, le cas échéant, des conditions générales de la licence souscrite par votre établissement. Toute autre reproduction ou représentation, en tout ou partie, sous quelque forme et de quelque manière que ce soit, est interdite sauf accord préalable et écrit de l'éditeur, en dehors des cas prévus par la législation en vigueur en France. Il est précisé que son stockage dans une base de données est également interdit. 


\section{Téléphone mobile et expression identitaire : réflexions sur l'exposition technologique de soi parmi les jeunes}

Article inédit. Mis en ligne le 20 octobre 2010.

\section{Mahdi Amri \& Nayra Vacaflor}

Mahdi Amri est ATER, Docteur qualifié en Sciences de l'Information et de la Communication, IUT NancyCharlemagne, Université Nancy 2.

Nayra Vacaflor est ATER et Doctorante en Sciences de l'Information et de la Communication, Université Bordeaux 3 Laboratoire MICA (Médiations, Information, Communication, Arts), http://mica.ubordeaux3.fr.

Plan

Introduction

Téléphone mobile, prolongement corporel, affichage identitaire

Appropriation du téléphone mobile et technologisation de soi

Conclusion

Références bibliographiques

\section{RESUME}

Cet article est le fruit d'une enquête de terrain qui associe les entretiens à l'observation participante. Il présente un nouveau concept lié à l'usage du téléphone mobile parmi les jeunes : l'exposition technologique de soi. Il analyse les façons avec lesquelles les jeunes construisent leur identité au moyen d'un outil qui dépasse largement sa fonction basique de contact social. Cet article se penche sur ce que font les nouvelles générations de jeunes avec leurs mobiles. Au-delà d'un usage utilitaire, ils expérimentent avec cet appareil de nouvelles formes d'amusement, d'intimité, d'interaction et d'identification aux sphères d'appartenance.

\section{ABSTRACT}

This paper is the result of a fieldwork research's that associates interviews and participant observation. We present a new concept related to the use of mobile phone among young: the technological exposition of the self. We analyze the ways in which youth construct their identities by using this technology that overtake the basic function of social contact. This paper deals about uses of mobile in new generations. Beyond a practical use, they experiment with this device to discover new ways of amusement, intimacy, interaction and identification in the belonging circles.

\section{RESUMEN}

Este artículo es el fruto de una investigación de terreno que asocia entrevistas en profundidad y observación participante. Este, presenta un nuevo concepto ligado al uso del teléfono móvil entre los jóvenes : la exposición tecnológica del ser. Además, se analiza las maneras con las cuales los jóvenes construyen su identidad por medio de un instrumento que sobrepasa ampliamente su función básica de contacto social. Este artículo trata sobre los usos de los móviles en las nuevas generaciones. Mas allá de un uso utilitario, estas generaciones experimentan con este aparato nuevas formas de diversión, intimidad, interacción e identificación a las esferas a las que pertenecen. 


\section{INTRODUCTION}

Le téléphone mobile est devenu en peu de temps un outil indispensable en termes de communication et de relations interpersonnelles et un véritable phénomène de société au niveau mondial. A la fin des années 1990, son utilisation a connu une augmentation brutale jusqu'à une quasi-saturation du marché peu après $2000^{1}$. D'abord réservé à une élite sociale pour une utilisation professionnelle, il s'est répandu jusqu'à devenir le moyen de communication privilégié d'un grand nombre de personnes, notamment les jeunes. Ces dernières années, les technologies liées au mobile ont connu une évolution fulgurante et dès 2005 , le nombre d'utilisateurs a dépassé 2 milliards dans le monde. En France, durant la dernière décennie, le téléphone mobile est entré dans quasiment tous les foyers. Le taux d'équipement frôlait les 90\% de la population en 2008 (Lienard, 2009), ce qui place la technologie mobile devant la technologie fixe voire devant toutes les technologies médiatisées Sung-Do, 2006).

Le téléphone mobile, phénomène contemporain de communication interpersonnelle, s'inscrit dans le quotidien des jeunes, dans un espace-temps subjectif donnant lieu à des interactions qui sont plus que routinières, c'est-à-dire fortement parlantes de soi. Dans l'univers mobile, les jeunes font plus qu'émettre ou recevoir des communications, ils déversent des secrets et une part cachée de leur personnalité sur l'objet. Ce jeu d'intimité conduit les utilisateurs à un exercice permanent d'énonciation de soi et d'ouverture sur l'autre, dans une perspective authentiquement identitaire. Des travaux en sciences de l'information et de la communication se sont concentrés sur l'influence des médias sur les identités. Ces recherches sont nombreuses notamment auprès des populations jeunes et adolescentes. Par une analyse des enjeux identitaires et des discours des jeunes enquêtés, nous proposons une exploration de quelques «pratiques mobiles» juvéniles afin de répondre aux questions suivantes: que signifie rester physiquement attaché à son téléphone mobile et l'avoir toujours sur soi ? Comment le téléphone mobile est-il devenu pour les jeunes un miroir de l'identité personnelle et en quelque sorte une " technologie de soi »? Comment ces derniers manifestent-ils l'expression du soi à travers cet outil ? Comment l'usage subjectif de cette " prothèse corporelle » qu'est le mobile dans un cadre interactionnel restreint, produit-il la reconnaissance des uns par rapport aux autres ?

Nous avons essayé d'appréhender la technologisation de soi à travers le téléphone mobile dans le milieu des jeunes et de la contextualiser dans les évolutions incessantes de leur construction identitaire. Partant de l'hypothèse que le téléphone mobile dépasse sa fonction initiale de lien social, nous avons centré notre attention sur les façons d'être, de se conduire et de s'exprimer, dans le processus identitaire mobile. L'objectif de cette recherche était d'examiner les usages du mobile jusqu'à maintenant très peu étudiés à partir d'une enquête qualitative par entretiens. Les données recueillies ont permis de jeter un nouveau regard sur la dimension identitaire et expressive présente dans les nouvelles formes d'appropriation du mobile.

Nous avons dépouillé une quinzaine d'entretiens parmi les 30 conduits auprès d'une population jeune (18-28 ans), multi-ethnique, habitant la Communauté urbaine de Bordeaux (CUB). Nous nous sommes limités dans ce travail à analyser la matière provenant

${ }^{1}$ En France, nous comptons un peu plus de 58 millions de cartes SIM en service dont $68 \%$ sont liées à des forfaits. Si nous ramenons ce chiffre à celui du nombre de personnes vivant en France au ler avril 2009 (64,2 millions selon l'INSEE), nous obtenons un taux de pénétration de 90,7\%.

http://telecom.sia-conseil.com/index.php/etudes/le-marche-de-la-telephonie-mobile-en-france-en-2009version-mise-a-jour.
} 
de la ville de Pessac et de Bègles. Le choix a été porté sur ces deux villes en raison de leur grande mixité ethno-culturelle. La population à laquelle nous avons été confrontés, au cours de la réalisation de l'enquête, était majoritairement maghrébine (59\%), 25\% issue de pays européens et $16 \%$ du continent asiatique (ces pourcentages font référence à notre public enquêté : les jeunes de 18-28 ans).

La rencontre avec les jeunes enquêtés s'est faite par le biais de réseaux formels d'associations culturelles et de réseaux informels liés à des contacts personnels. Pour réaliser ces entretiens, nous sommes allés trouver les jeunes dans leur lieu de vie. Une enquête par entretien implique une écoute fine, de la patience, mais aussi de la passion envers l'objet de recherche. C'est par ce type de démarche que nous avons essayé de « rentrer dans l'esprit» des jeunes rencontrés afin de recueillir le plus authentiquement possible leurs paroles.

Cet article propose donc une réflexion sur les usages et les représentations narratives du téléphone mobile chez les jeunes. Conduit dans une perspective multidisciplinaire (communicationnelle, sociologique, anthropologique et esthétique), il analyse les aspects significatifs de l'usage personnalisé d'une technologie miniaturisée mais fortement éloquente et parlante de soi.

Pour aborder l'ensemble de ces points, cet article est divisé en deux grandes parties. La première introduit certains concepts théoriques sur le téléphone mobile en tant que continuité de l'individu en rapport avec la problématique identitaire. La seconde est consacrée à la technologisation de soi à travers l'appropriation du téléphone mobile et ce, au-delà d'une compréhension techno-centrée de l'outil.

\section{TELEPHONE MOBILE, PROLONGEMENT CORPOREL, AFFICHAGE IDENTITAIRE}

L'interactionnisme symbolique définit une identité comme le résultat d'un processus d'identification et celui-ci comme un construit social et un ensemble de traits associés à un type, à un rôle ou à un groupe social. En partant du postulat que les identités sont de nature symbolique, relationnelle et non essentialiste et que leur construction s'inscrit dans une dynamique culturelle, nous proposons dans le cadre de cet article, de comprendre l'identité à partir d'un triple référent : situationnel, individuel et narratif.

Il sera ainsi question de différentes identités. Par exemple, on parle d'une catégorie jeune, vieux, homme, femme, français, étranger etc. Ces catégories peuvent servir de label pour caractériser l'individu dans une interaction. Elles contribuent à structurer son «identité situationnelle ». L'identité narrative, selon Ricœur, évoque le récit structuré de vie grâce auquel l'individu définit ses caractéristiques (Ricoeur, 1990). Cette identité narrative est étroitement liée avec ce que Michel Foucault appelle les «technologies de soi » (Foucault, 2001), notamment le téléphone mobile. Elle se sert des outils pour produire un récit de vie. Ce dernier peut être compris comme un vecteur d'interaction et de communication pour les jeunes. Situer les identités jeunes dans cette configuration nous aide à comprendre leurs rapports. Le référent situationnel renvoie à l'idée d'espaces, de scénarii et de lieux qui donnent au sujet l'idée de ce qu'il est, ce qu'il a été et ce que sont les possibilités objectives de ses pratiques mobiles. Il pourrait être le lien entre l'espace et la représentation que le sujet se fait de lui-même, ainsi qu'entre les images que les autres se font de cette identité. Ces identités peuvent être des structures de crédibilité déterminantes dans l'environnement médiatique des jeunes. En d'autres termes, le lieu social détermine des itinéraires quotidiens dans lesquels s'établissent les relations avec le réseau social, la ville, les institutions, etc. 
Nous avons constaté que les jeunes se «situent» dans la configuration des différents espaces dont ils font partie, en incluant impérativement le téléphone mobile comme un prolongement corporel. «Moi où je suis, où je bouge, mon téléphone est avec moi » nous disait Khadija, 21 ans, lors d'un entretien. Le référent situationnel prend encore plus d'importance à partir du moment où l'outil technique s'avère être l'instrument avec lequel ils s'approprient les différents espaces.

L'existence de l'individu est étroitement liée à l'identité personnelle, au langage autoréférent et à l'action réflexive. Le sujet est considéré comme porteur d'un développement cognitif et émotif spécifique ou comme un sujet social et culturel. L'agencement du sujet social individuel se développe en différents scénarii. Les connaissances que le jeune a acquises dans sa vie jouent un rôle essentiel au niveau individuel. L'identité sexuelle de l'individu en détermine une autre, pour certains génétique, pour d'autres culturelle. L'âge est aussi un référent individuel dont la nature est à débattre. En dernier lieu, on peut mentionner l'ethnicité qui peut être mise en relation avec les conditions socioéconomiques et politiques quand on l'associe avec des groupes minoritaires. Ainsi, toutes ces notions liées au réfèrent individuel doivent se comprendre comme appartenant à un univers de cultures.

Nous avons considéré que le référent individuel jouait un rôle fondamental pour pouvoir comprendre le «sujet jeune». Cette approche nous a été indispensable pour nous imprégner du contexte, de l'agencement social et notamment l'entourage individuel. Quant à l'objectivation narrative, nous allons la comprendre comme les formulations concrètes et matérielles de l'identité qui se véhiculent dans le corps, dans le langage, les préférences, les styles ou dans la consommation culturelle. Celles-ci sont des marques visibles des institutions et des groupes que les acteurs incorporent dans une révision et une transformation constantes. Le récit identitaire permet à chacun de justifier sa réussite personnelle en termes culturels et sociaux. Nous pouvons déjà apercevoir qu'un des aspects principaux pour la reconnaissance de l'identité peut être lié aux ressources techniques. Ces identités que l'individu revendique, doivent pouvoir se justifier sous la forme d'un récit. A tout moment, lors d'une interaction, l'individu peut être questionné sur une identité affichée. A chaque identité correspond un récit, taillé sur mesure en fonction de la situation et de la compagnie que l'individu peut exposer si nécessaire (Frame, 2008).

Nous ne pouvons pas dissocier l'identité, du contexte qui la façonne, d'autant que nous nous intéressons à l'utilisation du téléphone mobile dans ses diverses formes, et à travers les pratiques et les productions auxquelles il donne lieu. Ollivier, en effet, mentionne que «les principaux moyens de produire à un niveau collectif de l'identification sont les productions culturelles »(Ollivier, 2007, p138). En ce sens, toutes les utilisations que le téléphone mobile offre, peuvent être comprises comme des produits culturels et ces utilisations vont représenter de nouvelles formes de récit d'une identité. Le jeune et son identité narrative vont varier selon son état d'esprit, par rapport aux circonstances qu'il traverse. Pour nous, il ne faudrait pas séparer ces traits dans la compréhension de la construction d'une identité. Ce triple référent nous donne les bases principales pour essayer de comprendre le «sujet jeune » dans son contexte particulier de valorisation de soi.

Nous pouvons voir ainsi que les éléments de cette triple référence sont profondément liés entre eux. Néanmoins, ces distinctions proposées ne prétendent pas résoudre le problème de la construction identitaire. Elles nous conduisent à identifier plusieurs aspects de cette problématique permettant la compréhension du "phénomène jeune » et l'utilisation du mobile. 


\section{Le téléphone mobile prolonge-t-il I'individu?}

Plusieurs études ont souligné que pour la génération des jeunes et des adolescents il y a une nécessité certaine d'inventer des croyances, des valeurs et des signes d'orientation. David Le Breton, par exemple, justifie cette nécessité par un manque de traditions, de chemins tracés ou d'idéologies qui donneraient sens à leurs vies (Le Breton, 2008). Comment le téléphone mobile s'insère-t-il dans ce processus de construction identitaire? Comment le jeune s'exprime t-il à travers cet outil ?

En fait, le mobile ne représente pas seulement un objet pratique destiné à communiquer mais un objet surinvesti de sens et jouant un rôle essentiel dans la subjectivité réflexive de la personne. D’un point de vue démographique, le mobile touche le plus la catégorie des adolescents et des jeunes. Pour ces derniers, il est plus qu'indispensable, il est présent en tout lieu et à tout moment. Il représente un motif indissociable des représentations de la vie contemporaine (AFOM, 2007). Par sa présence massive et ses multiples incorporations à la personne (dans la poche, entre les mains, dans le sac, sous l'oreiller), le mobile acquiert aux yeux de son possesseur un intérêt particulier et l'accompagne dans tous les lieux et circonstances de vie. Le mobile, cette petite boite précieuse n'a pas pour seule fonction la communication pure. Il s'est intégré dans la gestualité contemporaine du téléphoniste mobile en devenant son ami intime et un remède efficace contre la solitude.

Les jeunes rencontrés montrent à quel point le mobile révèle l'identité de son utilisateur et combien les discours des usagers illustrent la thèse de l'énonciation de soi à travers les différentes stratégies de personnalisation de l'objet. Cela étant, il a été noté que ces jeunes qui personnalisent tous leurs téléphones, réfléchissent à ce que peut véhiculer cette pratique en termes d'images et de valeurs en rapport avec la problématique identitaire :

«Le téléphone c'est comme un complément de la personne, il est comme la personne, une partie de la personne, on essaie de l'habiller comme on s'habille, de le parer, de le rendre pareil comme nous ", Andrea, 20 ans

«Moi je le porte toujours, pour moi c'est rare de sortir sans le porter », Eko, 28 ans

«Par contre je sais que pour certains Français c'est comme un jean ou des chaussures une partie de leur corps quoi, un truc esthétique qui va avec le corps qui va avec les vêtements, qui ne va pas différencier un jean ou un T-shirt, des chaussures ou des dernières lunettes, je voudrais dire c'est une partie de l'esthétique vestimentaire, donc ça fait partie en quelque sorte du corps, quoi », David, 24 ans

«Mon portable pour moi c'est toute ma vie. Tu peux trouver tout un catalogue de ma vie dedans. Je suis fière de le dire. J'aime mon portable...j'aime » Inès, 23 ans

Dans l'univers des jeunes, le mobile occupe une place privilégiée parmi les technologies du quotidien. Au sein de ces technologies, le mobile se trouve dans un espace, un temps et des mobilités très changeants. Le corps humain s'active et se transforme tous les jours à travers une multitude d'actions (manger, marcher, se laver, se vêtir...) et le mobile n'échappe pas à cette gamme de gestes routiniers mais indispensables pour toute personne. Les jeunes, transformant leurs mobiles avec une temporalité propre, s'approprient un espace, essaient de se retrouver dans les sociétés mondialisées. Ils expérimentent une émancipation à leur manière propre et authentique à l'égard de ce média. Il est donc nécessaire de repenser le concept d'identité à l'égard du mobile et de la communication, non pas seulement comme un ensemble de données ou caractéristiques dont l'origine est dans le passé, mais surtout comme une construction dynamique qui se produit dans le croisement entre de multiples interpellations qui proviennent de divers espaces et temporalités. 
Dans la culture juvénile, le téléphone mobile est devenu un objet irremplaçable, comme d'ailleurs les accessoires vestimentaires. Le personnaliser n'est donc pas considéré comme un artifice, mais plutôt comme une action spontanée et impulsive. Le mobile est un prolongement de la tenue vestimentaire et sortir de son domicile sans l'emporter est comme sortir nu, ou tout au moins en ayant oublié de mettre un vêtement ou un accessoire de base : les clés, le pull, le pantalon, les chaussures, etc. Restant souvent à portée de main, il se laisse transformer par son utilisateur. Ce dernier ne cessant de «l'esthétiser » et de le personnaliser en fonction de ses goûts, en fait un réservoir de son intimité, un peu à la manière du journal personnel et des carnets de voyage qui constituent un espace privilégié pour l'exposition « technologique» de soi.

Le corps est un médium puissant de communication. Vêtements, accessoires, ornements, déguisements l'ont toujours soumis aux codes d'une esthétique partagée. Déshabillé, habillé, le corps mis en forme permet à l'individu de s'inscrire dans une société, rentrer dans une communauté, appartenir à un groupe: "le corps dit qui nous sommes et qui on voudrait être au regard d'autrui" (Caron et Caronia 2005, p291). Leopoldina Fortunati souligne : "Bien que la perte de naturalité soit une caractéristique naturelle de l'être humain, le corps a toujours conservé, du moins jusqu'aux temps modernes, une certaine distance à l'égard des machines, dès lors qu'on le considérait comme l'expression même de la naturalité. Un éventuel mélange du corps avec les machines, expression suprême de l'artificialité, aurait été jugé tout à fait impossible à proposer. Cela ne signifie pas que le corps n'a jamais pactisé avec l'artifice. Au contraire, le corps ne parle que s'il est habillé d'artifices. Du maquillage, aux tatouages, des scarifications aux mutilations, des opérations de chirurgie esthétique à l'habillement, tous ces éléments concourent à transformer de manière visible le corps en corps social» (Fortunati, 1998, p87).

En effet, l'esthétique vestimentaire communique beaucoup sur la personne. Les vêtements qu'on porte l'été sont nettement différents de ceux de l'hiver, la tenue du travail n'est pas celle du domicile, s'habiller d'une manière ou d'une autre est tributaire de plusieurs paramètres. A travers le vêtement, chacun fournit des informations concernant son référent individuel: identification sexuelle, position socioprofessionnelle, origines ethniques, attitude envers la société, caractère, personnalité et état d'esprit (Fortunati, 1998, p90). De même, à mesure que les jeunes changent l'habillage de leur téléphone mobile, ils s'inscrivent dans une logique identitaire (habiller son mobile du drapeau national, accrocher une clé USB à l'objet ou un bâton de rouge à lèvres pour certaines filles).

Les entretiens et les témoignages précités montrent que, pour les jeunes, le téléphone mobile fonctionne à la manière d'une affiche de soi. Ils s'y reconnaissent et s'en servent comme un instrument de réassurance, permettant de maintenir sa confiance en soi, comme en témoigne par exemple le réflexe de vérifier si on le porte toujours sur soi. Le téléphoniste mobile apparaît comme englouti par son appareil chaque fois qu'il le tâte, dans une sorte de liaison obsessionnelle. Cet abandon de soi nous fait spontanément penser à plusieurs scènes du film Matrix dans lesquelles le héros Nero est commandé par son mobile et même, tombe sans résistance dans les filets de la machine (Matrix, film de science-fiction écrit et réalisé par les frères Wachowski, 1999).

\section{Le mobile et l'angoisse de la séparation}

Peu d'objets technologiques de l'ère contemporaine se sont glissés de manière aussi massive et significative dans la gestualité quotidienne. Par ses promesses, ses spécificités et son utilité technique, le mobile se laisse facilement manipuler. Il est aisé de l'adapter au corps en établissant une correspondance harmonieuse entre corps et objet. Les discussions 
avec les jeunes étayent ce constat. Ces derniers n'ont pas hésité à décrire leur attachement solide au mobile, allant même jusqu'à évoquer un véritable rapport affectif :

«Moi par exemple j'arrive pas à me passer de mon portable quand je l'oublie à la maison je sens que quelque chose me manque, comment dire : Ah j'ai oublié mon portable! Il faut revenir pour chercher mon portable! C'est comme si je vais perdre contact avec le monde extérieur, voilà, ou bien je vais à un certain moment me sentir seule, parce que une fois je n'ai rien à faire je commence à biper les gens, à m'amuser, écrire des SMS, une fois je suis sans portable je commence à me sentir seule, il y a quelque chose qui me manque, voilà je pense que je suis séparée du monde extérieur », Rita, 24 ans

"Moi je dors avec mon téléphone, aujourd'hui j'ai une heure pour dormir et j'ai dormi comme ça avec le téléphone, parce que il y a toujours quelque chose qui peut être importante », Natacha, 24 ans

«Pour moi il est tout le temps avec moi, dès que je rentre chez moi je le mets dans un endroit proche pour justement l'entendre, tout le temps presque je manipule le téléphone...c'est impossible que je laisse le portable chez moi, mon portable il faut toujours qu'il soit avec moi et surtout je vérifie souvent la batterie, du coup j'ai acheté un chargeur pour la voiture, pour pouvoir le charger dans la voiture ! », Wajih, 28 ans

«En sortant la première chose à laquelle je pense est ce que j'ai sur moi mon portable tandis qu'on peut sortir sans radio, sans walkman, mais le portable il doit être là, sachant que je ne vais pas avoir d'appels mais je dois l'avoir avec moi », Sawsan, 28 ans

«Il est toujours avec moi, je l'utilise comme une montre, comme un agenda oui et je sais qu'avec le portable je suis toujours en contact avec les gens et parfois ça remplace mon interphone, j'habite au dernier étage et mes amis m'appellent, je leur jette les clefs pour ouvrir et ils viennent chez moi ! C'est essentiel de rester toujours en contact pour moi », Leila, 27 ans

«Mon portable ah oui mon portable je peux pas vivre sans mon portable. J'avais quatre mais c'est celui-là maintenant que j'ai. J'en ai au moins sept chez moi. Je peux pas vivre sans mon téléphone, il dort à côté de moi c'est sa place ici, il est si important pour moi. Le téléphone je pense à rien quand je l'oubli, il faut que même si je suis loin, je repars, je re-rentre à la maison pour le prendre, il fait partie de ma vie maintenant », Ibtissame, 21 ans

Ces propos sont révélateurs de la place qu'occupe le mobile dans la vie de chaque enquêté. Il est présent de manière privilégiée à tous les moments de la journée. Il crée un type sophistiqué de rapport physique entre sujet et objet. Ainsi, il n'est pas nécessaire de rappeler le premier réflexe adopté par les jeunes une fois sortis de leur domicile : vérifier de ne pas avoir oublié le mobile. Ce geste évident mais indispensable s'apparente au " geste de la poche » ou à une autre série de gestes liés au mobile : manipuler son téléphone, jouer et photographier avec, le gratter, le caresser, l'habiller ou même le laisser nu sans housse. Ce geste tend à produire chez la personne un sentiment de réassurance. S'il renvoie à une dimension méta-communicante de l'objet, il peut aussi suggérer un portrait caricatural du téléphoniste mobile une fois séparé de son appareil, s'imaginant être coupé du monde.

Quitter son domicile sans mobile équivaut à un état de désorientation totale. Loin d'être exagérée, cette interprétation est confirmée par les dires des jeunes eux-mêmes. Le téléphone mobile, considéré davantage comme un objet inséparable du corps que comme un outil de la communication, les accompagne dans tous les moments de la journée. Il est devenu, en quelque sorte, le réceptacle de leurs affects. En ce sens, la relation du sujet à l'objet peut être révélatrice de sa psychologie et de ses états d'âme. L'insécurité, le sentiment de solitude, de manque et de séparation de son réseau relationnel peuvent être quelques-unes des conséquences de la perte ou de l'oubli du mobile chez les jeunes 
«accros ». En effet, la perte de l'objet symbolise à la fois toutes ces choses. Elle participe au dysfonctionnement de tout un univers. C'est pour cela qu'on peut désormais considérer le mobile comme un objet qui ne cesse de nous lier.

\section{Dormir avec son téléphone mobile}

"Il est tout le temps avec moi », "moi je dors avec mon téléphone ", « il fait partie de ma vie ». Dans les entretiens, rien n'est plus naturel que d'être constamment en compagnie de son téléphone mobile. C'est devenu effectivement une «technologie du soi ». En fait, l'avoir toujours sur soi est synonyme de contact permanent avec le réseau, dans le cas contraire, la communication serait bloquée. Le mobile est souvent transporté dans tous les endroits imaginables : le lieu de travail, les transports, les pièces de la maison, au fur et à mesure des allées et venues, jusque dans la salle de bains ou la chambre à coucher. Le fait de dormir avec, n'est pas exceptionnel, parmi les jeunes, mais une quasi-constante. Il apparaît nécessaire de le garder toujours, même sous l'oreiller pour être certain de l'entendre au cas où il sonnerait la nuit. Ne pas l'avoir toujours avec soi se révèle comme un indice de non-appropriation.

L'habitude de dormir avec son mobile est intéressant pour l'analyse. Il démontre que cet appareil est devenu pour certains jeunes plus qu'un objet indispensable. Son intégration dans la routine corporelle étant un fait évident, l'objet est inséparable du sujet et l'éteindre un moment ne sera quasiment jamais pensé. Le mobile apparaît comme un prolongement corporel, dont l'usage relève de l'intime. Prolongement corporel ou seconde peau, le mobile l'est sans doute dans la mesure où "la frontière marquée entre intérieur et extérieur est un rôle joué habituellement par la peau» (Craipeau, 2007, 221), Cette image démontre combien l'objet peut représenter un véhicule de l'identité. La matérialité de l'objet est éloquente audelà de sa représentation sociale. Autrement dit, à travers l'action d'éteindre son mobile, c'est toute une dimension méta-communicationnelle de l'objet qui se laisse découvrir. L'objet fonctionne ainsi comme un producteur de notre existence, par conséquent il n'est pas inintéressant de remarquer jusqu'à quelle limite cette "technologie de soi » tisse des rapports fins et intimes avec le corps humain.

Corinne Martin souligne dans Le téléphone portable et nous quelques routines d'attachement au téléphone mobile. Elle cite: "Les consultations systématiques de l'écran à la sortie de classe ou de réunion sont monnaie courante. Dans le sondage, déjà cité, réalisé par l'IFOP pour Orange en 2003, le téléphone mobile est le second objet vérifié « automatiquement avant de quitter mon domicile le matin " par $17 \%$ des Français, certes bien après les clés de maison (62\%) mais avant le portefeuille (16\%). De la même façon dans l'enquête, bien peu oublient leur téléphone le matin. » (Martin, 2007, p112).

Les jeunes ont la spécificité d'évoquer l'incorporation à l'objet avec le langage le plus simple et familier. Parce qu'à plusieurs moments des entretiens, il leur a été demandé de décrire leurs sentiments face à l'oubli ou la perte de leur téléphone, ils n'ont pas hésité à se montrer prolixes en révélant leurs rituels associés au téléphone mobile. «En sortant la première chose à laquelle je pense est celle de ne pas avoir oublié mon portable tandis qu'on peut sortir sans radio, sans walkman, mais le portable doit être là ». Cette incorporation de l'objet, cette proximité et cette familiarité avec le corps n'ont rien de rationnel et ne suscitent par conséquent aucune réflexivité. Il est à noter que la petite taille du mobile facilite son déplacement devient un élément essentiel pour son incorporation. C'est ce que soulignent les paroles du vice-président de Nokia-France : "le succès du téléphone dépend de sa petitesse et de sa légèreté, pour le mettre dans la poche» (Martin, 2007, p112). Cependant, cette caractéristique matérielle du mobile n'est pas la seule à nous intéresser. C'est également sa polyvalence dans la mesure où il est utilisé de plus en plus comme 
téléphone, horloge, baladeur, carnet personnel, boîte à jeux ou même outil pour navigation qui explique les mécanismes profonds d'attachement à l'objet. Cette polyvalence transforme l'objet en un monde à soi.

\section{APPROPRIATION DU TELEPHONE MOBILE ET TECHNOLOGISATION DE SOI}

En resituant le téléphone mobile dans le contexte social des cinq dernières années, on s'aperçoit vite qu'aujourd'hui, c'est un objet que peuvent acquérir des personnes à revenus économiques très différents. Le fait d'en posséder ne signifie pas qu'il existe une différence en terme de statut social avec celui qui n'en possède pas ou qui en possède un moins performant. Le mobile ne représente plus un critère de distinction sociale. Yucef (27 ans) et Sofie (20 ans), deux jeunes interviewés, arrivent dans ces lignes à exprimer le même constat.

Yucef: «Moi je dirais que non, parce que généralement on peut trouver ceux qui ont le moins de moyens généralement, on trouve. Ici en France par exemple tu vas dans les banlieues, les gens que tu croises, normalement ce sont ceux qui ont le moins du moins ce sont ceux qui ont les plus jolis portables alors que ceux qui sont suffisamment riches ne s'attardent pas sur les détails, les petits trucs »

Sofie : « Mais justement pour ça, ceux qui veulent montrer qu'ils s'en foutent du portable et qui veulent montrer que ça ne les intéresse pas particulièrement, justement ils ont de beaux portables, alors que ceux qui n'ont pas les moyens et qui veulent montrer qu'ils sont bien habillés, cool et tout, ils prennent des portables, donc ça caractérise l'image qu'ils veulent donner d'eux-mêmes. »

Dans cet extrait, la vieille question de l'être et du paraître est explicitement abordée. C'est le paradoxe du comédien gréco-romain qui dissimule son identité sur la scène, en jouant, en simulant, en portant un masque. Le protagoniste est loin de tromper le spectateur, il est sincère et tout en jouant son rôle, il ne manque pas de rester lui-même. Il est celui qui représente pertinemment une identité et qui s'approche au mieux de son public. Dans l'espace privé/public des jeunes, ces derniers immergent l'entourage dans la scène de communications mobiles en transformant des séquences conversationnelles ordinaires en "séquences théâtralisées»(Goffman, 1974, p145). Les questions que les interlocuteurs peuvent se poser sont les suivantes: quel est le degré d'implication de l'acteur dans la scène ? Dans quelle mesure a-t-il conscience de donner un spectacle ? Le comédien réussitt-il, dans cette représentation transmise au public à rester lui-même tout en se mettant dans la peau d'un autre? Le spectateur devrait être conscient que le jeune utilisateur ne dissimule pas son identité authentique mais qu'il est justement en quête d'une reconnaissance sociale à travers une représentation.

\section{Une signification renversée de l'acquisition du mobile}

Au-delà de la signification renversée de la possession de l'objet, nous pouvons distinguer deux catégories symboliques : la première pour laquelle exposer le mobile n'est plus un signe de richesse dans une société où toute personne est susceptible d'en avoir au minimum un. La deuxième pour laquelle, le mobile, surtout tactile, devient un objet à forte connotation sémiotique : par exemple, la dernière vague des tactiles a plusieurs caractéristiques techniques $(3 \mathrm{G}$, logiciels d'exploitation, mémoire très large, jeux interactifs, visioconférence, etc.). On se retrouve là face à des mobiles avec des propriétés techniques et valeurs polysémiques toujours augmentées.

Cette distinction relative à une technologie de soi très populaire à l'heure actuelle, fait partie de tout un répertoire de comportements. Chez les jeunes, avec le tactile, nous 
pouvons constater une mise en scène constante du téléphone high-tech, justement pour révéler une chose importante : "Toi t'as un tactile, et ben moi aussi !». Il s'agit d'avoir accès aux mêmes services, d'être aligné sur les pairs, bref de posséder équitablement un objet high-tech, pour concrétiser le besoin d'être ensemble. Dans un sens plus large, cette idée d'être ensemble se manifeste dans l'usage et l'appropriation de toutes les technologies de communication. Ces dernières sont là pour réduire l'espace-temps des utilisateurs en multipliant les opportunités de contacts (Licoppe, 2000).

De même, les jeunes ont tendance à exhiber continuellement leur téléphone afin de se forger une certaine image sociale (supériorité et pouvoir technologiques). Ce double spectacle du visible/invisible, être/paraître joué par les deux catégories, met en évidence l'une des facettes étranges de l'objet mobile. Ce dernier est devenu non seulement un signe du jeu opéré sur la distinction sociale, mais aussi et surtout un écran derrière lequel l'on se retire périodiquement (Hall, 1971) pour énoncer ce qu'on souhaite être aux yeux des autres.

Dans la vie sociale, les actes relationnels exigent une certaine mise en forme. Les activités les plus courantes ne laissent pas au corps un rôle purement instrumental : regards, mimiques, attitudes corporelles sont autant de signes que les interlocuteurs engagés dans une relation utilisent et reçoivent en tant que tels. Erving Goffman, pour décrire la représentation de soi, adopte des modèles empruntés du théâtre. L'individu, en présence d'autrui, ordonne les expressions qu'il produit et contrôle les impressions que les autres reçoivent par des techniques que cet auteur assimile aux techniques de mise en scène (Caune, 1997). L'exemple des utilisateurs exhibitionnistes du mobile relève parfaitement de ce type de techniques mises en place pour la théâtralisation de soi.

En effet, dans Mobile attitude: ce que les portables ont changé dans nos vies, Alban Gonord et Joëlle Menrath soulignent : "Si la société peut être depuis toujours être considérée comme une vaste scène où chacun joue plus ou moins consciemment un rôle, le mobile y promène depuis quelques années son petit théâtre itinérant. De nouvelles scènes prennent vie sous nos yeux, faisant naître de nouveaux gestes, de nouveaux comportements, de nouvelles façons aussi de jouer avec les autres personnages et les codes en vigueur. Quittant le point de vue privilégié de l'intériorité, on se place alors dans la peau du spectateur. » (Gonord et Menrath, 2005, p149).

En cherchant dans les récits, nous avons trouvé un exemple qui nous montre comment le marketing mobile réussit à aiguiser davantage les désirs technologiques des jeunes. Délia et Ibtissame avaient comme projet de changer leur mobile "pourri » contre un téléphone tactile. Elles ont trouvé une offre séduisante : "votre tactile à 1 euro. » Il est important de souligner qu'avec de telles offres le nombre des abonnés dépasse celui des prépayés. L'AFOM, dans son rapport sur le marché de la téléphonie mobile indique que la France est l'un des pays européens les mieux positionnés en terme d'abonnés (AFOM, 2008). En effet, Délia et Ibtissame ont toutes les raisons pour s'abonner: mobile 3G, forfait économique, SMS illimités ainsi que des appels gratuits les soirs et les week-end. Au milieu des années 90, avec l'essor de la téléphonie mobile, l'acquisition d'un mobile avait une dimension symbolique forte dans la mesure où elle conduisait à la valorisation de l'image publique. L'utilisation du mobile en public attirait l'attention de l'entourage, faisait sortir un peu de l'anonymat, et conduisait chacun à assumer ou refuser involontairement cette distinction créée. Maintenant, avec la possession généralisée du mobile, ce n'est presque plus le cas.

Ce qui nous paraît important ici, ce n'est pas l'acquisition de l'objet par ces jeunes. mais plutôt le constat que, pour retrouver des significations partagées liées à la possession de l'objet, qu'il soit performant ou non, il est nécessaire de s'écarter de l'objet lui-même pour s'intéresser aux comportements qu'il génère. Comme tous les techno-objets (walkmans, 
baladeurs, micro-ordinateurs) qui, au début de leur diffusion, étaient précieux puis ont perdu de leur valeur une fois popularisés, le téléphone mobile a une force et une fonction de modèle. Au début de son lancement, les anecdotes sur les façons de l'exposer, les tentatives de le voler, les craintes de le faire tomber en panne, étaient très courantes et dessinaient les traits d'une comédie technologique dont les personnages étaient assimilés à de faux «branchés". Même si ces 5 dernières années, l'exposition du mobile est statistiquement moins fréquente, la mise en scène à laquelle il donne lieu dans la vie quotidienne est toujours là. Montré ou caché, le mobile continuera toujours de se dénuder sous l'œil public.

«Pour moi le design de l'appareil photo peut refléter beaucoup de personnalité, si la personne préfère quelque chose de pratique entre les hommes on peut trouver parfois le modèle de téléphones qui sont plus pratiques et pour les femmes je ne sais pas c'est plutôt le design qui est beau... ", Julia, 23 ans

«Moi il me faut toujours que mon portable soit à l'égard de mon être. Bonne qualité d'appareil photo, stockage musique, enregistreur et beaucoup de mémoire » Khalid, 17 ans

Avec leur mobile, bien loin des performances esthétiques et fonctionnelles, les jeunes instaurent un rapport purement affectif. Le mobile, en effet, se transformant dans leurs mains en une technologie de soi, s'infiltre dans leur vie. Il y trouve place dans des moments où eux-mêmes sont instables et en train de se chercher. Le mobile joue ainsi le rôle d'un compagnon fidèle de leur train de vie, de leur existence faite de hauts et de bas. Nous retrouvons à nouveau la notion situationnelle et individuelle de l'identité d'un jeune. Parce qu'il fait partie des éléments essentiels de son espace, l'utilisateur du mobile est conduit à en prendre soin, à lui accorder plus ou moins d'attention, selon les humeurs et les occasions du moment :

« Nous changeons tout le temps les images dans notre portable...parce que tout le temps nous prenons de photos et chaque jour est différent donc il faut toujours renouveler notre personnalisation », Delia, 23 ans.

«Moi je ne change pas toutes mes personnalisations...cela dépend de l'émotionnel, du moral, comment ça va aujourd'hui, est ce que ça va bien ou ça va pas bien ? Roland, 33 ans.

\section{Construction de soi et stratégies mobiles de personnalisation}

Nous avons tous hérité des manières correctes qui permettent de vivre ensemble et structurent nos modes de socialisation, comme par exemple le fait de rester discret, de ne pas espionner les autres, de veiller à ne pas perdre ses affaires... Avec le mobile, la logique de ces «bonnes manières » est totalement renversée. Dans un contexte «intra-tribal », dès que des copains se mettent à comparer leurs mobiles au niveau des sonneries, des marques, des couleurs, de toutes performances esthétiques ou pratiques, on s'aperçoit que chacun peut reconnaître facilement le téléphone de l'autre à partir d'un simple petit détail. Ce n'est pas seulement le son, la forme ou même la marque qui peut être identique, ce sont aussi des détails pointus qui aident les acteurs à établir les correspondances entre l'objet et le soi : rayures sur le dos, brillance, tags, etc. Bien sûr ces correspondances n'apparaissent pas ex nihilo, elles ne sont pas inhérentes au mobile mais appartiennent au référent situationnel de l'acteur.

En effet, s'il n'y avait pas de tels liens d'amitié ou de telles affinités entre ces jeunes, le mobile ne jouerait pas son rôle d'identification. Le mobile n'est pas comme un malin génie qui nous divulguerait des secrets sur l'identité inconnue des gens. Il pourrait sonner toute la journée dans un milieu sans que personne ne s'en aperçoive! Le téléphone mobile est 
un objet inscrit dans le prolongement de connexions sociales organisées. Son identification aux « personnages » est liée à un réseau fin mais complexe de relations. Mais ce n’est pas le nombre de personnalisations qu'on a effectuées sur le mobile qui va aider à le retrouver, une fois caché ou perdu, dans un environnement proche. C'est le référent situationnel en jeu qui va permettre d'identifier le propriétaire du mobile. La présence des mobiles dans l'espace partagé est soumise à un code de conventions et à un capital de reconnaissance mutuelle. Cette question du cadre plutôt que de l'objet lui-même nous montre que le mobile n'est pas un objet comme les autres. Plus qu'un autre "il est au centre, au carrefour des identités et des relations partagées, plus qu'un autre il est au plus près du vécu et des habitudes de chacun.» (Gonord et Menrath, 2005, p114).

\section{Un outil personnel, une technologie de soi}

En fait, si le téléphone mobile est conçu comme un puissant indicateur de l'identité individuelle, il révèle beaucoup de choses sur soi et sur l'autre. L'existence de l'individu est étroitement liée à la construction d'un soi, l'expression d'une identité, ainsi qu'à la sensibilité expressive. Les actions qu'il entreprend à un niveau personnel sur son mobile, engendrent de nouvelles passions et satisfactions, de nouveaux pouvoirs. Toutes celles que les jeunes réalisent avec leur téléphone s'articulent complètement avec leur «soi », leur identité. Il n'existe aucune action humaine qui ne dépende pas d'un cadre général de répétition. L'homme organise sa vie active et intellectuelle selon des «technologies" historiques, dérivées de discours interprétatifs de la réalité qui ne sont construits ni pour lui ni pour un autre. Foucault (Foucault, 2001) mentionne que les technologies du soi sont un ars vivendi : elles concrétisent les opérations qu'un individu doit exécuter sur son corps et son âme pour aboutir à un état complet d'humanité. Nous allons transposer cette analyse auprès des jeunes pour comprendre comment ils fabriquent et s'approprient le mobile comme une technologie de soi dans son environnement quotidien.

Les jeunes adoptent divers modes d'utilisation de cet outil. Le mobile n'est plus un téléphone lié à un espace précis (bureau, maison, transports). Il est devenu un instrument de communication indépendant de tout espace. C'est un téléphone qui se réfère exclusivement à l'individu. La communication est devenue un atout dans toutes les actions que le sujet peut mener avec l'outil. Elle se démocratise et s'individualise pour toutes les personnes qui acquièrent un mobile.

Plant (Plant, 2008), dans une étude transculturelle, mentionne que les adolescents japonais utilisent leurs « keitai » (mobile en langage familier) pour mener une vie plus libre, loin de la surveillance des parents. Le même auteur mentionne que les mobiles permettent aux prostituées de Bangkok de faire des deals avec des clients potentiels d'une manière plus indépendante. Le mobile est à l'origine une série de changements dans les sociétés qui nous invitent à repenser et à revisiter le concept du soi.

Le téléphone mobile, pour les jeunes, est devenu un outil personnel par excellence. Il appartient à un «soi », et ce « soi » est à la fois le «sujet en tant qu'objectivation produite par des dispositifs normatifs et travaillés par le réseau complexe des rapports de pouvoir, et une subjectivité qui se réapproprie d'elle-même [...] et qui simultanément se réinvente, se produit. » (Revel, 2005, p209). Autrement dit, les jeunes prêtent au mobile différentes fonctions et valeurs par rapport aux situations. Ils peuvent créer, par exemple, des échelles de valeurs : intimité-« extimité » (Tisseron, 2001, visible- invisible, être- paraître.

Cette technologie du soi ouvre aux jeunes plusieurs compétences sociales, graphiques et textuelles dont ils se servent au quotidien. Ainsi la «production » d'un soi est inscrite dans une temporalité linéaire et permanente chaque fois que le possesseur regarde l'heure, vérifie s'il n’y a pas des appels manqués, ou un SMS ou tout simplement s'il est là...C'est 
dans cette mesure que l'on peut dire que les rapports entre cet outil et le soi, sont produits subjectivement. Cela veut dire qu'ils possèdent le privilège de fonder des réactions qui seront entre autres inventives, inauguratrices, créatives. De plus, avec la rapidité d'accès et les capacités de stockage du mobile, les possibilités de mise en scène du soi sont devenues illimitées.

\section{Expression identitaire «mobile»}

Le jeune s'inscrit dans un réseau social et acquiert une multiplicité de comportements, de significations diverses, au sein de la collectivité. C'est là « où il recrée et fait de la diversité et de la polysémie dans l'interaction sociale» (Martin-Barbero, 1990). Connaître les manières et les sens que les jeunes établissent à partir de l'utilisation de mobiles, c'est comprendre pourquoi, comment, quand, où et dans quel but ils créent et récréent des relations culturelles en utilisant les SMS, les appels, la visioconférence, l'Internet mobile...

La communication à travers le mobile et la circulation de SMS avec leurs propres codes linguistiques et paralinguistiques s'insère dans les sphères sociales d'usage. De même que les sonneries ou la variété des musiques que le mobile offre, nous renseignent sur une utilisation propre à un individu, les jeunes utilisateurs personnalisent ces éléments en réactualisant un "conflit social ", c'est-à-dire, en créant une distinction symbolique d'usage et d'appropriation de codes. Avec leurs besoins de communication et d'appartenance, ils revendiquent en quelque sorte une vie "privative " au sein d'un ordre public. Le mobile s'inscrit dans l'histoire des dispositifs techniques comme un point de rencontre entre deux filières de services, celle de la téléphonie fixe et celle des outils de communication privées. En agrégeant dans une même technologie les fonctions de mobilité, la communication et l'expression graphique, le mobile reconfigure d'une part, les pratiques antérieures de communication orale et propose d'autre part, des modalités relationnelles différentes de communication.

Ces nouvelles opportunités tiennent au lien étroit que le mobile permet d'établir entre production identitaire et organisation du système relationnel. Les jeunes $\mathrm{y}$ expriment, sous formes variées, certains traits de leur identité afin de mettre cette production au service de la sélection, de l'entretien et de l'enrichissement de leur propre répertoire. Dans ce sens, ces produits configurent ce que les jeunes sont. Selon les termes de Kaufman, ils se produisent dans des subjectivités et dans des processus de "génération constante où il n'y a plus d'institutions qui déterminent la façon absolue de la formation de sujets. » (Kaufman, 2005). C'est l'énonciation du soi qui se met ici au centre de la scène et marque sa place dans un espace mobile. Les institutions formelles sont mises à l'écart de cette configuration nouvelle des identités. L'énonciation du soi, la narration d'un soi, sont inscrites dans des singularités propres aux jeunes : marques émotionnelles, SMS, langage courant, verbes exprimant des sensations, musiques bruyantes, etc. Les contenus et les actions que ces jeunes réalisent avec le mobile sont fortement attachés à leur contexte.

Mais on ressent de plus en plus dans certains espaces sociaux, notamment dans les cultures juvéniles, le besoin de construire une image et de narrer une histoire. On est peut-être là devant des identités non homogènes et non excluantes. Elles sont une "construction qui se narre et c'est dans la diversité de ces narrations que les identités se construisent " (Martin-Barbero, 2001). Le lien entre narration et identité n'est pas seulement expressif, mais il est aussi constitutif et c'est dans la diversité des narrations que les identités se construisent.

Un premier exemple intéressant est celui d'Eko, une jeune étudiante chinoise, qui arrive à identifier le sexe de ses correspondants sur mobile, en écoutant les sonneries de son téléphone tout en restant dans la cuisine. Eko explique ainsi qu'elle a associé aux filles des sonneries douces et aux garçons des sonneries fortes. Un deuxième est celui de Touria, 
jeune fille africaine, qui attribue des sonneries musicales romantiques à sa petite famille : parents, frères, sœurs. Dans un troisième cas, Rita, jeune doctorante associe le numéro de ses parents à l'hymne national de son pays (Maroc). Enfin, Karim attribue de belles chansons aux personnes aimées, dans ses choix de mobile.

Parmi les jeunes interrogés, certains recourent à des stratégies très sophistiquées dans la personnalisation de leur mobile. Ainsi, Alejandro est devenu un expert de la personnalisation des sonneries. Pour reconnaître ses amis qui l'appellent sur son mobile il a associé des chansons de rap interprétées par sa communauté d'intimes. Il ne s'agit donc pas de suivre la démarche classique à savoir naviguer sur Internet pour télécharger des sonneries, mais de créer ses propres sonneries au-delà d'un simple transfert de fichiers commercialisés et ce faisant, de marquer ces sonneries de sa propre empreinte, de l'individualiser. De manière générale, l'investissement affectif est essentiel dans la stratégie de personnalisation. La référence à certains goûts par rapport au mobile n'est pas une finalité en soi. Il importe tout autant de distinguer l'horizon relationnel qu'elle laisse apparaître. Autrement dit, lorsque l'on associe les chansons que l'on aime à des personnes chères, on exprime notre amour de la musique choisie, mais aussi et surtout des personnes désignées. C'est un travail toujours recommencé, car si des sentiments vers une personne précise sont immuables, les façons de les exprimer sont en variation constante. Cette double action répond précisément à la question naïve - à quoi sert une sonnerie musicale associée à une personne ? - que se posent les gens depuis l'apparition du mobile et même après sa banalisation.

\section{CONCLUSION}

Le téléphone mobile ouvre de nombreuses perspectives de recherche, notamment dans ses rapports avec l'identité. Les différentes pratiques et les modes appropriation du mobile sont étroitement liés à l'identité des jeunes, le mobile est "le bric-à-brac de l'identité jeune». Il est devenu l'objet d'un attachement non pas seulement physique mais aussi affectueux. Le «soi » est représenté dans tous les moments, dans les situations, les actions et les expressions dans lesquels le mobile est impliqué. A la question de savoir comment le téléphone mobile est devenu pour les jeunes un miroir de l'identité, une des réponses suggérée est la suivante: le mobile est effectivement un objet réflexif de l'identité qui rassemble histoires individuelles et interactions constantes, dans la mesure où il est devenu un instrument qui parle authentiquement $d u$ «soi ».

Il existe à nos yeux une lutte identitaire qui se revendique au quotidien avec cet objet. Autrement dit, les jeunes défendent leur identité, leur autonomie dans la communication, leur sociabilité en se reformulant constamment par le biais du mobile. Cette démarche se fait au milieu d'échanges, de théâtralisations, de narrations de soi et de brouillages identitaires. Elle permet de découvrir une esquisse des pratiques sociales de ces jeunes et la manière dont ils intègrent le mobile dans leur vie indépendamment des potentialités techniques qui leur sont offertes. L'affirmation d'un soi n'a pas de sens sans autrui. Et cette affirmation et sa reconnaissance se fait au jour le jour, en interagissant avec les multiples offres symboliques que le monde leur livre.

Le téléphone mobile offre plusieurs possibilités d'appropriation, certes, mais le référent intériorisé s'avère être plus éloquent. Il se confronte à tous les moments de la vie individuelle des jeunes. L'exposition technologique que les jeunes pratiquent avec le mobile renvoie à leur condition humaine et à leur champ de liberté. Avec l'extension extraordinaire de ses potentialités techniques, d'une génération à une autre (G4, G5, G6...), le mobile permet de perpétuer la narration de soi et l'expression identitaire. La question qui se pose est celle de l'horizon vers lequel cette capacité immense d'énonciation 
de soi, facilitée par le mobile, conduit. Quel est le sens profond de ce «bazar d'intimité » que le téléphone mobile a créé autour de lui, dans le contexte subjectif d'usage, au point de devenir un logo de l'identité personnelle ? Qu'est-ce que le rapport homme-machine est en train de nous apprendre sur le soi, sur autrui ainsi que sur l'objet lui-même et les différentes corrélations possibles entre ces multiples entités ? 


\section{REFERENCES BIBLIOGRAPHIQUES}

AFOM, (2007), «Le téléphone mobile aujourd'hui. Usages et comportements sociaux », http:// www.afom.fr/v4/STATIC/documents/rapport_gripic_integrale.pdf (dernière consultation : 22 novembre 2009).

Caron, André, Caronia Letizia, (2005), Culture mobile: Les nouvelles pratiques de communication, Montréal, PUM.

Caune, Jean, (1997), Esthétique de la communication, Paris, PUF.

Craipeau, Sylvie, (2007), « Le corps en jeu », http:/ /www.instituttelecom.fr/archive/174/Livre_vert_version_web.pdf T, p.221 (dernière consultation : 20 décembre 2009).

Fortunati, Leopoldina, (1998), «Revêtir des technologies », Réseaux, n90.

Foucault, Michel, (2001), Technologies du soi, Dits et écrits, vol. II (1976-1988), Paris, Gallimard.

Frame, Alexander, (2008), Repenser l'interculturel en communication. Performance culturelle et construction des identités au sein d'une association européenne, thèse en Sciences de l'Information et de la Communication, dirigée par Boutaud Jean-Jacques, université de Bourgogne.

Goffman, Erving, (1974), Les cadres de l'expérience, Paris, Minuit.

Gonord, Alban, Menrath, Joëlle, (2005), Mobile Attitude : ce que les portables ont changé dans nos vies, Paris, Hachette Littératures.

Hall, T-Edward, (1971), La dimension cachée, Paris, Le Seuil.

Kaufman, Alejandro, (2005), "Educación y sociedad: transformaciones culturales y nuevas subjetividades", Curso de Posgrado Enseñanza de las Ciencias Sociales: construcción de conocimiento y actualización disciplinar, FLACSO Argentine.

Le Breton, David, (2008), Cultures adolescents. Entre turbulence et construction de soi, Paris, Autrement.

Licoppe, Christian, (2000),"Issues about the uses of information technologies : going beyond the network metaphor and starting back from actual patterns of embodied and mediated interactions", http://www.limsi.fr/Individu/turner/DCP/Paris2000/licoppePcd.pdf (dernière consultation : 16 octobre 2009).

Lienard Fabien, (2009), «L'avenir du SMS en marketing mobile », Actes du 1er colloque international Nouveaux médias : nouvelles technologies pour un nouveau monde, Université de Bahreïn.

Martin-Barbero, Jesús, (1990), “De los medios a las prácticas”, in Cuadernos de Comunicación y Prácticas Sociales, UIA, México, ${ }^{\circ} 1$.

Martin-Barbero, Jesús, (2001), Al sur de la modernidad. Comunicación, globalización, multiculturalidad, Pittsburgh, IILI.

Martin, Corinne, (2007), Le téléphone portable et nous : En famille, entre amis, au travail, Paris, L'Harmattan.

Ollivier, Bruno, (2007), Identité et identification. Sens, mots et techniques. Paris, Lavoisier Hermès. 
Plant, Sadie, (2008), On the Mobile: The Effects of Mobile Telephones on Social and Individual Life. Libertyville, IL: Motorola,

http://www.motorola.com/mot/doc/0/234_MotDoc.pdf, dernière consultation : 14 décembre 2009.

Revel, Judith, (2005), Expériences de la pensée, Paris, Bordas.

Ricour, Paul, (1990), Soi-même comme un autre, Paris, Seuil.

Sung-Do Kim, (2006), "Quelques remarques sur l'écologie culturelle de la technologie mobile », http://www.inst.at/trans/16Nr/07_1/sung-do16.htm, 16 septembre 2006 (dernière consultation : 13 décembre 2009).

Tisseron, Serge, (2001), L’intimité surexposée, Paris, Ramsay. 